\title{
3 Research Soure \\ Identification of potential prognostic markers for osteosarcoma by integrated analysis
}

\section{Yuxiang Ge}

Department of Orthopedic Surgery, Minhang Hospital, Fudan University

Wang Ding

Department of Orthopedic Surgery, Minhang Hospital, Fudan University

Chong Bian

Department of Orthopedic Surgery, Minhang Hospital, Fudan University

Huijie Gu

Department of Orthopedic Surgery, Minhang Hospital, Fudan University

Jun Xu

Department of Orthopedic Surgery, Minhang Hospital, Fudan University

Xiaofan Yin ( $\square$ xiaofanyinfdu@126.com)

Fudan University

\section{Research article}

Keywords: Osteosarcoma, Weighted gene co-expression network analysis (WGCNA), Prognosis, Hub gene identification

Posted Date: August 19th, 2020

DOI: https://doi.org/10.21203/rs.3.rs-52760/v1

License: (c) (i) This work is licensed under a Creative Commons Attribution 4.0 International License.

Read Full License 


\section{Abstract}

Background: Osteosarcoma (OS) is the most common type of musculoskeletal malignant tumor, accounting for over $30 \%$ of primary skeletal sarcomas. Although great efforts have been made, the mechanism of OS still remains largely unknown. In this study, we aim to identify gene modules and representative candidate biomarkers for clinical diagnosis of patients with OS, and reveal the mechanisms of OS progression.

Methods: Weighted gene co-expression network analysis (WGCNA) was conducted to construct a coexpression network and investigate the relationship between modules and clinical traits. Functional enrichment analysis was performed on module genes. Protein-protein interaction (PPI) network was constructed to identify the hub gene and the expression level of hub genes was validated based on another dataset.

Results: A total of 9854 genes were included in WGCNA, and 17 gene modules were constructed. Gene module related with OS in sacrum was mainly enriched in skeletal system development, bone development and extracellular structure organization. Furthermore, we screened the top 10 hub genes and further validated 5 of the 10 (MMP13, DCN, GNG2, PCOLCE and RUNX2), the expression of which were upregulated as compared with normal tissues.

Conclusion: The hub gene we identified show great promise as prognostic markers for the management of OS and our findings also provide new insight for molecular mechanism of OS.

\section{Introduction}

Osteosarcoma (OS) is the most common primary malignant tumor of bone, typically affecting the long bones of the limbs in adolescent and elderly individuals. OS commonly affects the long bones of the limbs. The most common sites are the femur, the tibia, and the humerus. Other likely locations include the skull, the jaw and the pelvis $[1,2]$. OS can be classified into several different sub-types associated with varying degrees of aggressiveness. Most OSs are high-grade malignancies, which is characterized by its aggressiveness and strong tendency for the development of pulmonary metastases [3]. The 5-year survival rate for patients with metastatic disease or recurrences is less than $25 \%$, indicating an extremely poor prognosis. Therefore, a better understanding of the underlying molecular mechanisms involved in the tumorigenesis and prognosis of OS is critical to actively develop novel therapeutic targets $[4,5]$.

Previous studies using microarray data of OS have conducted differentially expressed genes (DEGs) analysis between metastatic and non-metastatic samples and functional enrichment analysis [6]. Several DEGs such as epidermal growth factor receptor (EGFR) and signal transducer and activator of transcription 3 (STAT3) were explored to function in the progression of OS [7, 8]. However, these analyses cannot reveal the connections and interactions among genes that are crucial in biological processes. 
Weighted gene co-expression network analysis (WGCNA) has been proved to be an effective method to reveal the correlations between genes and phenotypes in different samples [9]. WGCNA can be used to identify gene modules of highly correlated genes, to construct a weighted gene co-expression network, and to correlate gene modules with external sample traits. As a result, WGCNA has been widely used to help discover candidate biomarkers or therapeutic targets in various biological processes including cancer [10-12].

In this study, we aimed to construct co-expression module using the expression data of osteosarcoma and find the modules of co-expressed genes which affected specific sites of OS. The GO and KEGG enrichment analysis were performed on the interested modules to analyze the involved cell signal pathways and functional genes, which would help in understanding the main function of genes in specific modules. Furthermore, the module gene network was constructed and identified the hub genes in the module by Cytoscape software. Finally, we validated the top values of genes in the PPI network based on another data set and got 5 hub genes, such as MMP13, DCN, GNG2, PCOLCE and RUNX2, which may be related to the clinical characteristics and development of OS. Our study not only laid a theoretical foundation for further research and the discovery of new OS target biomarkers, but also helped to build a new promising therapeutic system for OS in clinical.

\section{Materials And Methods}

\section{Data acquisition and processing}

Series matrix files and data tables of the microarray platform for WGCNA searched with keywords "Osteosarcoma" (Title) AND "Homo Sapiens"(Organism) were obtained from NCBI Gene Expression Omnibus (GEO) (http://www.ncbi.nlm.nih.gov/geo) with accessing number GSE19276. The combined data set consist of a total of 47 osteosarcoma samples, including the tibial group $(n=13)$, femoral group $(n=18)$, pelvic group $(n=7)$, Sacral group $(n=2)$, rib group $(n=2)$ and calcaneus group $(n=5)$. Before WGCNA analysis, we first mapped the array probes to the respective gene IDs by applying the array annotations. Then we removed the probes with multiple genes from the dataset and calculated the average expression of the gene values measured by multiple probes. An appropriate threshold was determined based on different threshold of expression value. WGCNA algorithm was applied to evaluate the gene expression value. Furthermore, flashClust tools in $\mathrm{R}$ language were used to perform the cluster analysis of samples with appropriate threshold value $[9,13,14]$.

\section{Construction of WGCNA and identification of modules}

By constructing co-expression network gene modules associated with osteosarcoma, we applied function soft Connectivity in WGCNA package to construct a co-expression network of all genes in the GSE19276 dataset. The top 5000 genes were screened by this algorithm for further analysis with the soft threshold set at 9. The weighted co-expression relationships among the subjects in all data sets in the adjacency matrix was evaluated by paired Pearson correlations [9]. Then, a topological overlap matrix (TOM) similarity function was applied to transform the weighted adjacency matrix into a TOM to estimate the 
co-expression relationships between genes. Each TOM was used to perform hierarchical clustering analysis via the flashClust function in R. Average linkage hierarchical clustering was used to construct a clustering dendrogram of the TOM matrix. Different branches of the clustering dendrogram represented different gene modules, and different colors represented different modules. Gene were classified based on their expression patterns and weighted correlation coefficients of genes. Genes with similar patterns are grouped into one module. Module-trait associations were estimated using the correlation between each module and clinical phenotype provided by GSE19276 dataset, which permits easy identification of expression patterns highly correlated to clinical traits of interest.

\section{Functional and pathway enrichment of gene modules}

To identify the biological functions and signaling pathways involved in modules, we performed gene ontology (GO) analysis and Kyoto encyclopedia of genes genomes (KEGG) pathway analysis using The Database for Annotation, Visualization and Integrated Discovery v6.8 (https://david.ncifcrf.gov/) and the threshold was set as $p<0.05[15,16]$.

\section{Hub Gene Analysis and Validation}

Hub genes have been shown to be functionally significant and highly interconnected with nodes in a module. In order to screen the top ranked genes with critical role in the protein-protein network (PPI), we construct PPI by uploading all genes in the selected module to the Search Tool for the Retrieval of Interacting Gene (STRING) database (http://www.string-db.org/) [17]. The Cytoscape software was further applied to perform the network analysis and the genes with top 10 degree were identified as hub genes [18]. Independent gene expression profiles (GSE9460) containing osteosarcoma and healthy bone tissue were obtained from the Gene Expression Omnibus and test set was used to validate the expression values of 10 genes with the highest degree. The dataset GSE9460 contained 15 primary osteosarcoma tissues and 3 healthy bone tissues.

\section{Results}

\section{Data processing}

To generate gene co-expression networks, a total of 47 tissue sample raw files of osteosarcoma data sets were downloaded from the GEO (http://www.ncbi.nlm.nih.gov/geo). The raw expression data were firstly subjected to Robust Multiarray Averaging (RMA) background correction and their processed signals were log2 transformed and normalized by quantile normalization. The 'affy' $R$ package were used to summarize median polish probesets. There were a total of 9854 probes for further WGCNA analysis after nsFilter processing.

\section{Construction of Weighted Gene Co-Expression Network of osteosarcoma}

The sample clustering dendrogram of osteosarcoma is shown in Fig. 1A and all samples were included in the clusters. The soft-power threshold $\beta$ was determined by scale independence and mean connectivity analysis of modules with different power values ranging from 1 to 14. As shown in Fig. 1B-C, $\beta=9$ and $\beta$ $=9$ were selected for further analysis of the osteosarcoma as the power for which the scale-free topology 
fit index reached 0.90 . Gene modules were then detected based on the TOM matrix and 17 modules were generated in osteosarcoma for further analysis and labeled 1-17. The largest module contained 1440 genes while the smallest contains 30 genes, and averagely, each module contained 294 genes. Interaction relationship analysis of co-expression genes was shown in Fig. 2A-B.

\section{Module-clinical trait correlations}

Identification of genes related to clinical trait is of great interest to elucidate the underlying mechanisms behind the clinical trait. In our study, the clinical parameters of osteosarcoma patients, including caocaneum, femur, pelvis, rib, sacrum and tibia were involved in the analysis. Modules with common expression patterns Interaction analysis of co-expression modules that were associated with particular traits were identified based on the correlation between module eigengene and clinic traits (Fig. 3A). The eigengene dendrogram and heatmap was used to identify groups of correlated eigengenes. As shown in Fig. 3B-C, the dendrogram indicates that the green module significantly associated with osteosarcoma clinical traits (sacrum). Besides, we plot a scatterplot of Gene Significance vs. Module Membership in the green module (Fig. 3D).

\section{Functional enrichment analysis of genes in interested modules}

In order to better understand the biological function of the 266 candidate module genes, we used the online database DAVID (https://david.ncifcrf.gov/), functional annotation tool for GO analysis, and the KEGG analysis used the R package of DOSE and the clusterProfiler for pathway enrichment analysis [19, 20]. Go enrichment analysis was performed on the genes in the selected green module [16]. As shown in Fig. 4, Genes in the green module was mainly enriched in G0:0001501 (skeletal system development), GO:0060348 (bone development) and G0:0043062 (extracellular structure organization).

\section{Module genes network analysis and hub genes identification}

To get a better understanding of the association between genes in the green module, a protein-protein interaction (PPI) network from the module gene based on WGCNA was constructed using Cytoscape software. The co-expression networks of top ranked genes for the selected green module as shown in Fig. 5. According to the STRING database, genes connected with more than 6 nodes were taken as hub nodes in the PPI network (MMP13, COL6A1, DCN, FAM20C, FBN1, GNG2, PCOLCE, PCOLCE2, RUNX2 and VCAN). The expression level of these hub genes was shown in Fig. 6A.

\section{Hub gene validation}

The expression of genes with the top 10 values were validated using the GSE9460 data set (Fig. 6B). In the validation set, we found that 5 of the 10 genes (MMP13, DCN, GNG2, PCOLCE and RUNX2) were significantly up-regulated in osteosarcoma group $(p<0.05)$, consistent with the analysis of GSE19276.

\section{Discussion}

Osteosarcoma is the most common human primary malignant bone tumor. It usually occurs in children and adolescents with a high incidence, rapid progression, and great metastatic potential. Over the past decades, despite the rate of local tumor control has been improved with advances in the treatment 
strategies, the long-term survival rate for patients remains unchanged [1, 2]. So as to improve the overall survival rate of OS patients, a better understanding of the underlying molecular mechanism of OS is needed. Previous studied have demonstrated several target biomarkers of OS based on both gene expression profiling and micro-RNA profiling of the primary tumor [21]. Wang and Baumhoer et al. had analyzed the OS sample by RT-PCR or immunohistochemistry and observed that insulin-like growth factor-1 receptor (IGF-1R) and cysteine-rich intestinal protein 1(CRIP1) were independent prognostic markers for OS and the high expression of these two markers was correlated with progression and metastasis of OS [22, 23]. A study performed by Duan et al. evaluated the role of miRNA in the progression of OS by RT-PCR and reported that three miRNAs, miR-127-3p, miR-199a-3p and miR-376c, were significantly downregulated in OS cell lines compared to osteoblasts, whereas miR-191 and miR151-3p were increased in OS cell lines [24]. However, there's still a lack of effective prognostic biomarkers for patients with OS. WGCNA, an effective method to detect the complicated relationships between different genes and phenotypes, provides insights into signaling networks which might be associated with clinical traits of interest. The analysis of WGCNA allows for identification of biological-relevant modules and hub genes, which may eventually serve as biomarkers for detection or treatment [9]. By constructing WGCNA to detect gene expression of OS, Fan et al. identified matrix metallopeptidase 3 (MMP3) and vascular endothelial growth factor B (VEGFB) as critical genes for OS development [25]. Likewise, Wang et al. reported the involvement of Matrix extracellular phosphoglycoprotein (MEPE) and Hemoglobin A2 (HBA2) in OS [26]. However, the precise mechanism of OS remains to be elucidated and more candidate biomarkers or therapeutic target needs to be discovered. Here, we utilized WGCNA to investigate co-expression modules related to OS and predict candidate gene sets that underlies a given biological process.

A total of 17 co-expression modules were first constructed by the 9854 genes from 47 human OS samples by WGCNA method, followed by module-trait correlations. As a result, we identified the green module that related to clinical traits (patient's OS location). Then the functional enrichment analysis was conducted in green module. Consistent with the previous study by Xiong and colleague, our result showed that the green module was mainly enriched in pathway associated with skeletal system development, bone development and extracellular structure organization [6]. Since the development of OS was accompanied by the abnormal expression of oncogenes and anti-oncogene, the enriched pathway that the green module involved in may regulate genes participated in the development of the skeletal system. Heng et al. assessed the DEGs and microRNAs in OS samples and found the downregulated DEGs COL4A1 and COL5A1 were enriched in extracellular structure organization as well as extracellular collagen biosynthesis pathway [27]. Increased extracellular collagen degradation might promote the development of OS and metastasis to lungs, which indicated that genes involved in collagen synthesis might have a negative effect on extracellular matrix, which may be responsible for OS tumor invasion in an undirect manner.

In addition, we constructed PPI network and identified hub genes in OS-related regulatory network. Here we used a degree to evaluate the importance of genes in the regulatory network. The genes with the highest degrees were identified as hub genes and the top 10 hub genes were COL6A1, MMP13, DCN, 
FAM20C, FBN1, GNG2, PCOLCE, PCOLCE2, RUNX2 and VCAN. Furthermore, we validated 5 genes, MMP13, DCN, GNG2, PCOLCE, and RUNX2, had significantly high expression profile in the validation data set.

Several of the genes have been reported associated with OS. Matrix metalloproteinase-13(MMP13) has been identified as a critical modulator involved in tumor osteolysis. Ma et al. identified MMP-13 as the target gene of proteolytic enzyme that regulated tumor-induced osteolysis [28]. Hirahata and colleagues showed that high MMP13 expression was of great importance for the survival of OS cells [29]. Decorin (DCN), Procollagen C-proteinase enhancer protein (PCOLCE) and Runx2 were found to play a vital role in lung metastasis of OS $[30,31]$. Runx 2 is a kind of protein that play major roles in bone formation. A study by Won evaluated the expression of Runx2 by immunohistochemical staining of OS tissues and concluded that high expression of Runx2 was significantly correlated to OS metastasis. Overexpression of Runx2 was also responsible for poor response to chemotherapy relative to good responders [32]. No reports have revealed that G Protein Subunit Gamma 2 (GNG2) was related to OS and further study was needed.

\section{Conclusion}

To sum up, the green module was regarded as the most important module, which was significantly associated with progression of OS patients. Furthermore, the critical hub genes within the module were further validated by another dataset, which may serve as the potential diagnostic and prognostic biomarkers of OS.

\section{Abbreviations}

WGCNA: weighted gene co-expression network analysis; OS: osteosarcoma; PPI: protein-protein interaction; DEGs: differentially expressed genes; EGFR: epidermal growth factor receptor; STAT3: signal transducer and activator of transcription 3; GEO: Gene Expression Omnibus; TOM: topological overlap matrix; GO: gene ontology; KEGG: Kyoto encyclopedia of genes genomes; STRING: Search Tool for the Retrieval of Interacting Gene; RMA: Robust Multiarray Averaging; RT-PCR: Reverse TranscriptionPolymerase Chain Reaction; ME: module membership.

\section{Declarations}

\section{Acknowledgments}

We greatly appreciate the financial support from the National Natural Science Foundation of China (81772433).

\section{Funding}

This work was supported by grants from the National Natural Science Foundation of China (no. 81772433). 
Availability of data and materials

The datasets used and/or analyzed during the current study are available from the corresponding author on reasonable request.

\section{Ethics approval and consent to participate}

Not applicable.

\section{Patient consent for publication}

Not applicable.

\section{Authors' contributions}

Conception and design: Yuxiang Ge, Xiaofan Yin.

Collection and assembly of data: Yuxiang Ge, Wang Ding, Chong Bian.

Data analysis and interpretation: Yuxiang Ge, Wang Ding, Chong Bian, Huijie Gu, Jun Xu.

Manuscript writing: Yuxiang Ge

Final approval of manuscript: All authors.

\section{Competing interests}

The authors declare that they have no competing interests.

\section{Consent for publication}

Not applicable.

\section{References}

1. Klein MJ, Siegal GP. Osteosarcoma: anatomic and histologic variants. Am J Clin Pathol. 2006;125(4):555-81.

2. Wang LL. Biology of osteogenic sarcoma. Cancer J. 2005;11(4):294-305.

3. Kempf-Bielack B, Bielack SS, Jurgens H, Branscheid D, Berdel WE, Exner GU, et al. Osteosarcoma relapse after combined modality therapy: an analysis of unselected patients in the Cooperative Osteosarcoma Study Group (COSS). J Clin Oncol. 2005;23(3):559-68.

4. Conrad EU. ORTHOPAEDIC ONCOLOGY. DIAGNOSIS AND TREATMENT2009.

5. Kansara M, Teng MW, Smyth MJ, Thomas DM. Translational biology of osteosarcoma. Nat Rev Cancer. 2014;14(11):722-35. 
6. Xiong Y, Wu S, Du Q, Wang A, Wang Z. Integrated analysis of gene expression and genomic aberration data in osteosarcoma (OS). Cancer Gene Ther. 2015;22(11):524-9.

7. Pahl JH, Ruslan SE, Buddingh EP, Santos SJ, Szuhai K, Serra M, et al. Anti-EGFR antibody cetuximab enhances the cytolytic activity of natural killer cells toward osteosarcoma. Clin Cancer Res. 2012;18(2):432-41.

8. Fossey SL, Liao AT, McCleese JK, Bear MD, Lin J, Li PK, et al. Characterization of STAT3 activation and expression in canine and human osteosarcoma. BMC Cancer. 2009;9(1):81.

9. Langfelder P, Horvath S. WGCNA: an R package for weighted correlation network analysis. BMC Bioinformatics. 2008;9(1):559.

10. Wang H, Liu J, Li J, Zang D, Wang X, Chen Y, et al. Identification of gene modules and hub genes in colon adenocarcinoma associated with pathological stage based on WGCNA analysis. Cancer Genet. 2020;242:1-7.

11. Yin L, Cai Z, Zhu B, Xu C. Identification of Key Pathways and Genes in the Dynamic Progression of HCC Based on WGCNA. Genes (Basel). 2018;9(2):92.

12. Zhou XG, Huang XL, Liang SY, Tang SM, Wu SK, Huang TT, et al. Identifying miRNA and gene modules of colon cancer associated with pathological stage by weighted gene co-expression network analysis. Onco Targets Ther. 2018;11:2815-30.

13. Wan Q, Tang J, Han Y, Wang D. Co-expression modules construction by WGCNA and identify potential prognostic markers of uveal melanoma. Exp Eye Res. 2018;166:13-20.

14. Ni Y, Zhang Z, Chen G, Long W, Tong L, Zeng J. Integrated analyses identify potential prognostic markers for uveal melanoma. Exp Eye Res. 2019;187:107780.

15. Dennis G, Jr., Sherman BT, Hosack DA, Yang J, Gao W, Lane HC, et al. DAVID: Database for Annotation, Visualization, and Integrated Discovery. Genome Biol. 2003;4(5):P3.

16. Ashburner M, Ball CA, Blake JA, Botstein D, Butler H, Cherry JM, et al. Gene ontology: tool for the unification of biology. The Gene Ontology Consortium. Nat Genet. 2000;25(1):25-9.

17. Zhu Y, Bian Y, Zhang Q, Hu J, Li L, Yang M, et al. Construction and analysis of dysregulated IncRNAassociated ceRNA network in colorectal cancer. J Cell Biochem. 2019;120(6):9250-63.

18. Wisniewski N, Cadeiras M, Bondar G, Cheng RK, Shahzad K, Onat D, et al. Weighted Gene Coexpression Network Analysis (WGCNA) Modeling of Multiorgan Dysfunction Syndrome after Mechanical Circulatory Support Therapy. The Journal of Heart and Lung Transplantation. 2013;32(4):S223.

19. Yu G, Wang LG, Yan GR, He QY. DOSE: an R/Bioconductor package for disease ontology semantic and enrichment analysis. Bioinformatics. 2015;31(4):608-9.

20. Yu G, Wang LG, Han Y, He QY. clusterProfiler: an R package for comparing biological themes among gene clusters. OMICS. 2012;16(5):284-7.

21. Evola FR, Costarella L, Pavone V, Caff G, Cannavo L, Sessa A, et al. Biomarkers of Osteosarcoma, Chondrosarcoma, and Ewing Sarcoma. Front Pharmacol. 2017;8:150. 
22. Wang YH, Han XD, Qiu Y, Xiong J, Yu Y, Wang B, et al. Increased expression of insulin-like growth factor-1 receptor is correlated with tumor metastasis and prognosis in patients with osteosarcoma. $J$ Surg Oncol. 2012;105(3):235-43.

23. Baumhoer D, Elsner M, Smida J, Zillmer S, Rauser S, Schoene C, et al. CRIP1 expression is correlated with a favorable outcome and less metastases in osteosarcoma patients. 2011;2(12):970.

24. Duan Z, Choy E, Harmon D, Liu X, Susa M, Mankin H, et al. MicroRNA-199a-3p is downregulated in human osteosarcoma and regulates cell proliferation and migration. 2011;10(8):1337-45.

25. Fan H, Lu S, Wang S, Zhang S. Identification of critical genes associated with human osteosarcoma metastasis based on integrated gene expression profiling. Mol Med Rep. 2019;20(2):915-30.

26. Wang JS, Wang YG, Zhong YS, Li XD, Du SX, Xie P, et al. Identification of co-expression modules and pathways correlated with osteosarcoma and its metastasis. World J Surg Oncol. 2019;17(1):46.

27. Heng L, Jia Z, Bai J, Zhang K, Zhu Y, Ma J, et al. Molecular characterization of metastatic osteosarcoma: Differentially expressed genes, transcription factors and microRNAs. Mol Med Rep. 2017; 15(5):2829-36.

28. Ma O, Cai WW, Zender L, Dayaram T, Shen J, Herron AJ, et al. MMP13, Birc2 (CIAP1), and Birc3 (cIAP2), amplified on chromosome 9, collaborate with p53 deficiency in mouse osteosarcoma progression. Cancer Res. 2009;69(6):2559-67.

29. Hirahata M, Osaki M, Kanda Y, Sugimoto Y, Yoshioka Y, Kosaka N, et al. PAI-1, a target gene of miR143 , regulates invasion and metastasis by upregulating MMP-13 expression of human osteosarcoma. Cancer Med. 2016;5(5):892-902.

30. Wang S, Zhong L, Li Y, Xiao D, Zhang R, Liao D, et al. Up-regulation of PCOLCE by TWIST1 promotes metastasis in Osteosarcoma. Theranostics. 2019;9(15):4342-53.

31. Shintani K, Matsumine A, Kusuzaki K, Morikawa J, Matsubara T, Wakabayashi T, et al. Decorin suppresses lung metastases of murine osteosarcoma. Oncol Rep. 2008;19(6):1533-9.

32. Massi D, De Nisi MC, Franchi A, Mourmouras V, Baroni G, Panelos J, et al. Inducible nitric oxide synthase expression in melanoma: implications in lymphangiogenesis. Mod Pathol. 2009;22(1):2130 .

\section{Figures}




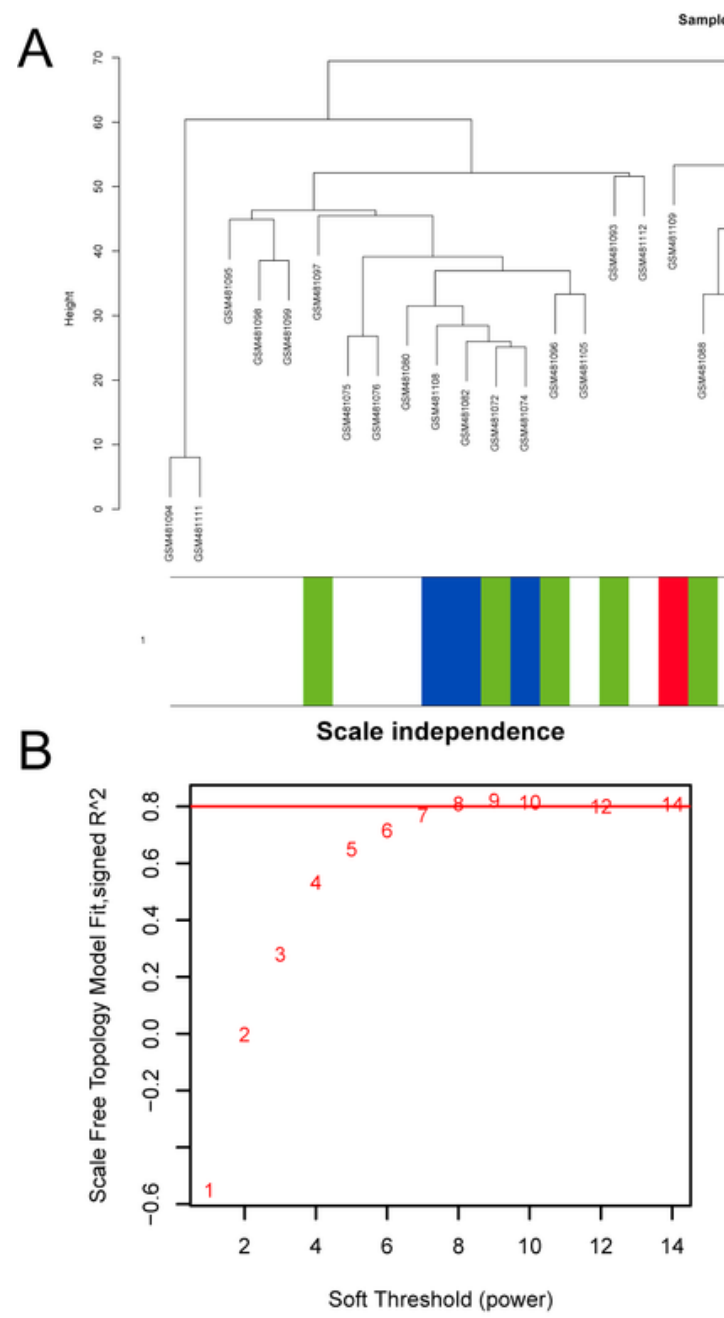

D

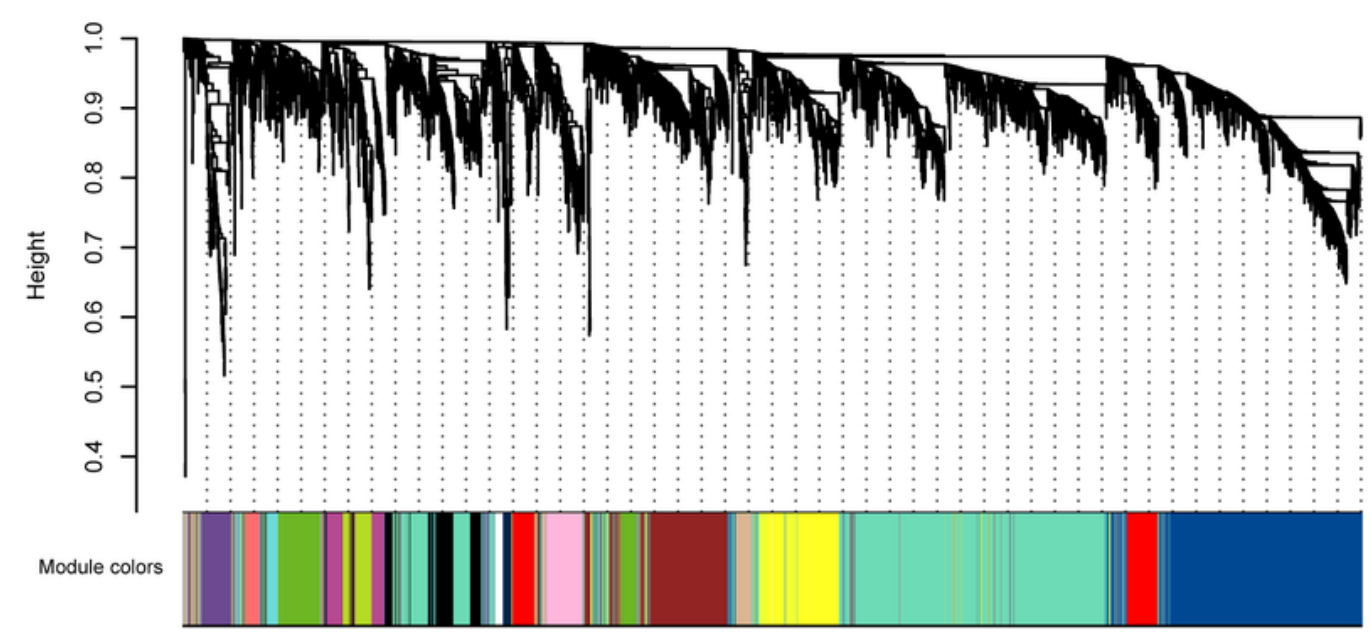

\section{Figure 1}

Gene modules identified by Weighted gene co-expression network analysis (WGCNA). (A) Hierarchical clustering of hub genes in the clustering analysis. Branches of the dendrogram represent together hub genes that are correlated positive. (B-C) Network topology of different soft-thresholding powers. The left panel (B) displays the influence of soft-thresholding power (x-axis) on scale-free fit index(y-axis). The right panel (C) shows the influence of soft-thresholding power (x-axis) on the mean connectivity (degree, 
$y$-axis). (D) Clustering dendrograms of GSE19276. The dissimilarity was based on topological overlap. The $y$-axis is the distance determined by the extent of topological overlap

A

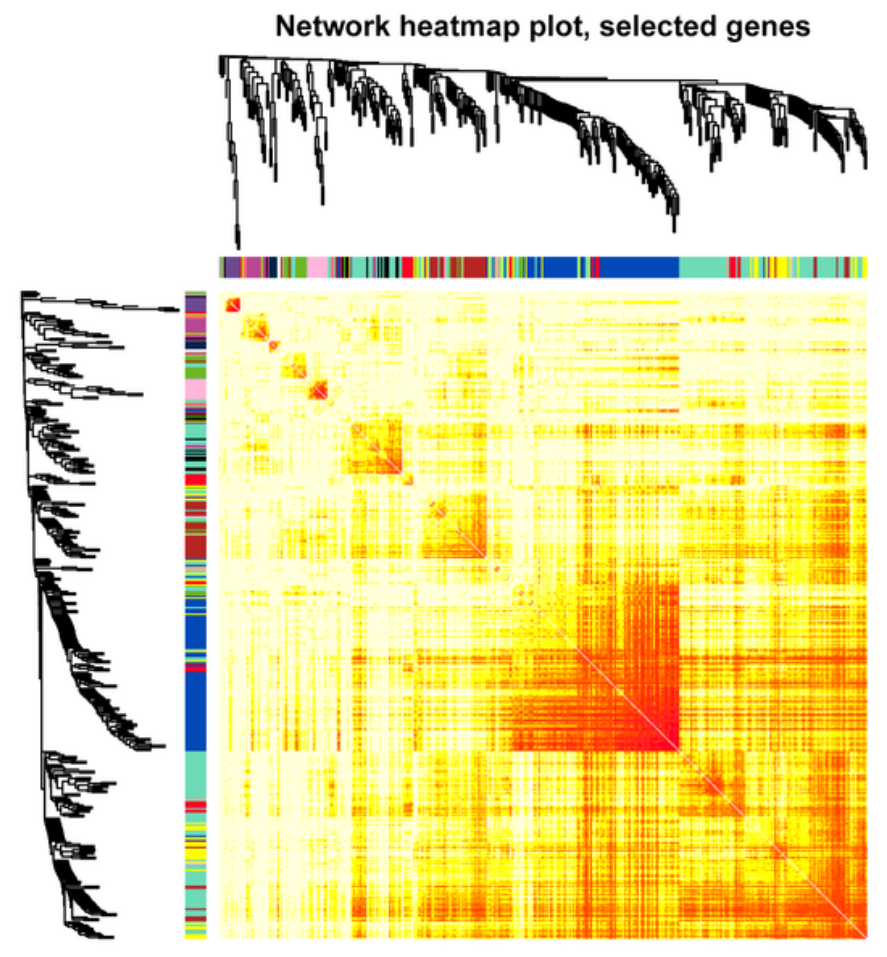

B

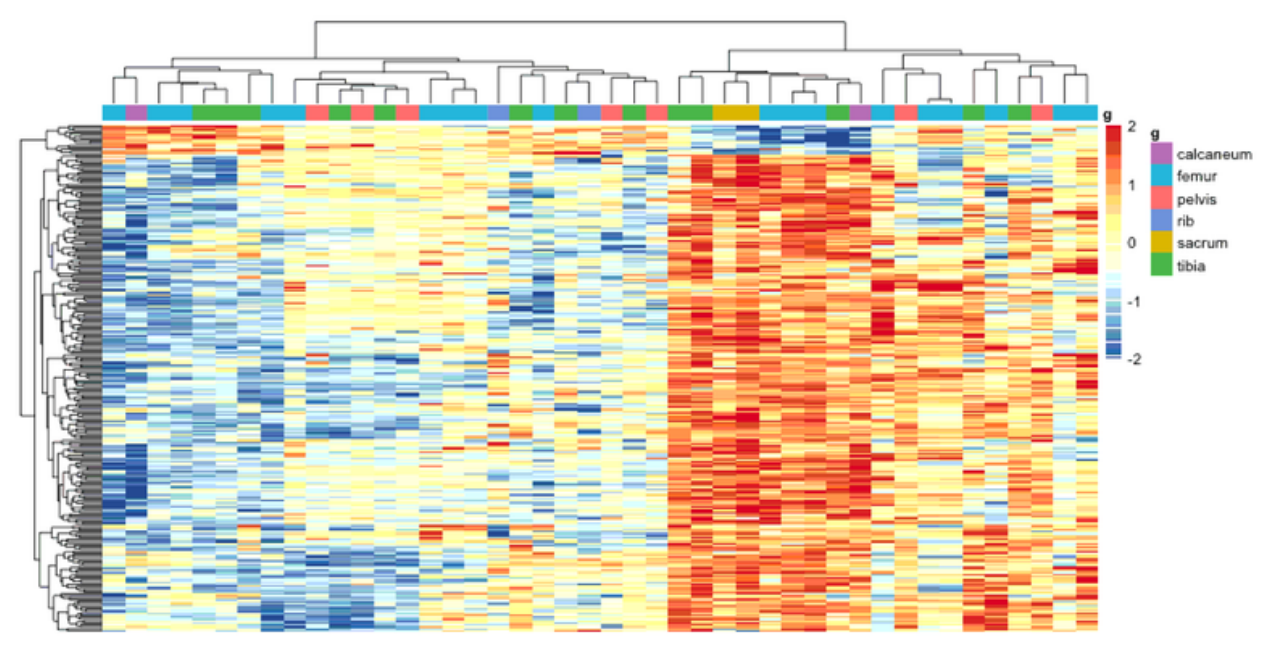

Figure 2

Visualizing the gene network using a heatmap plot. (A) Heatmap plot of topological overlap in the gene network. In the heatmap, each row and column correspond to a gene, light color denotes low topological overlap, and progressively darker red denotes higher topological overlap. Darker squares along the diagonal correspond to modules. The gene dendrogram and module assignment are shown along the left and top. (B) Heatmap of the difference of genes expression in the GSE19276. Red color represents upregulated genes while the blue represents the downregulated genes. 
A

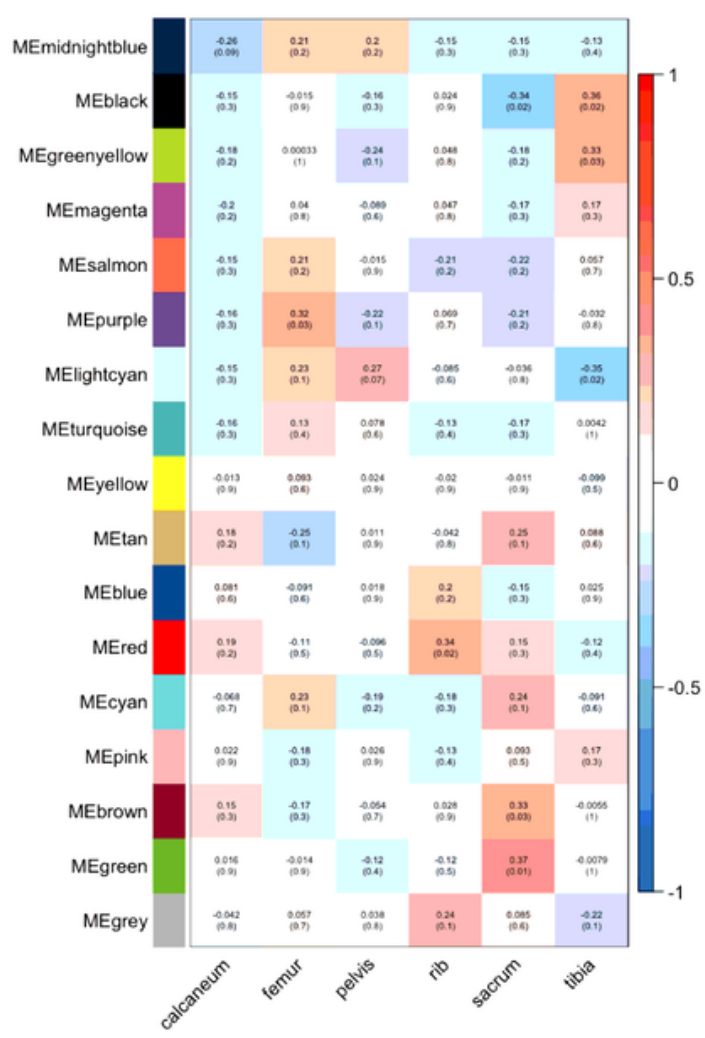

B

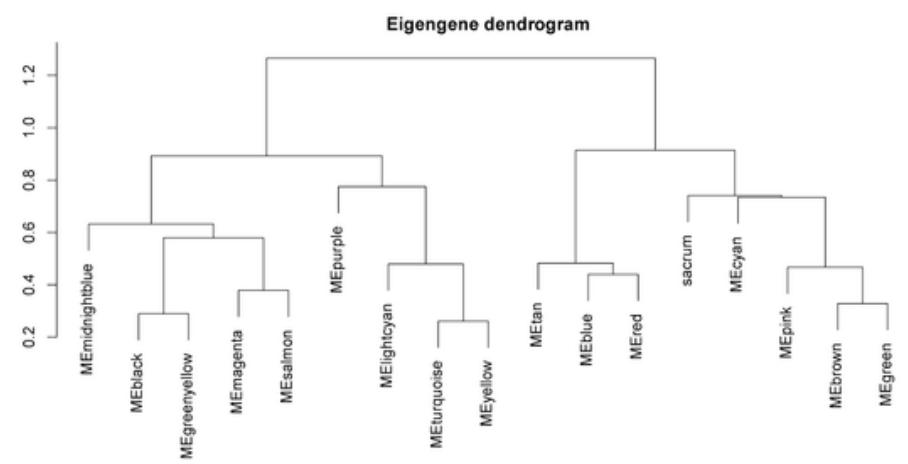

C

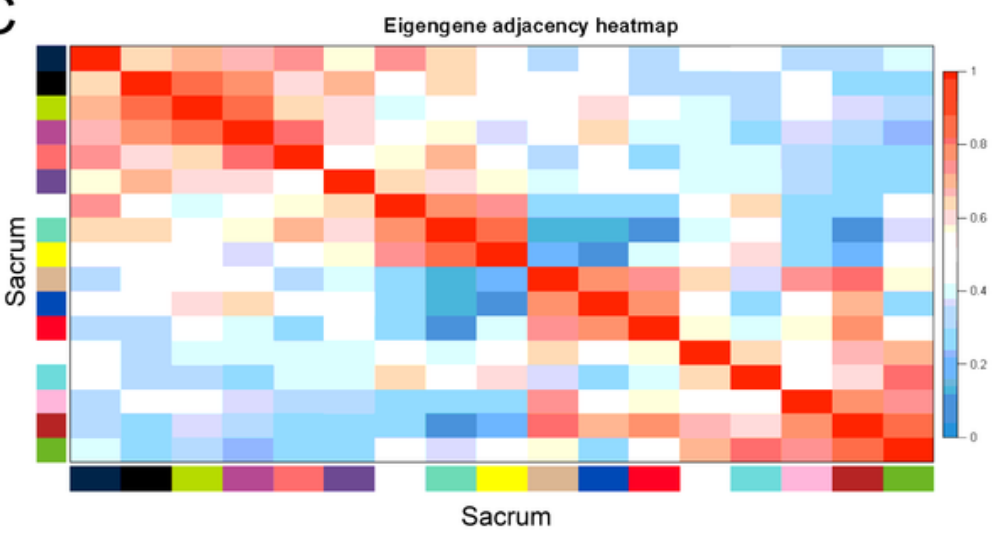

D

Module membership vs. gene significance cor $=0.36, p=2.1 e-09$

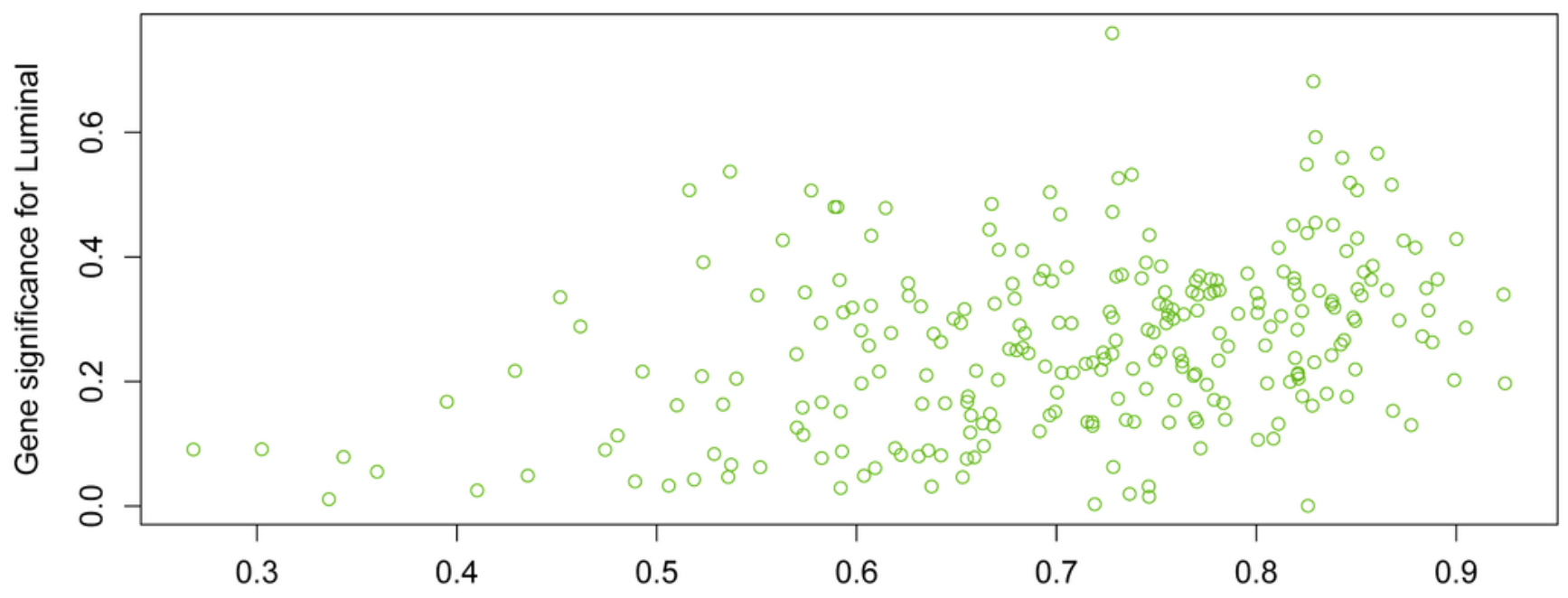

Module Membership in green module

\section{Figure 3}

Correlation between module eigengenes and clinical trait of OS. (A) Module-trait relationships. Each row corresponds to a module eigengene, column to a trait. Each cell contains the corresponding correlation and $p$-value. The table is color-coded by correlation according to the color legend. (B) Hierarchical clustering of module hub genes that summarize the modules yielded in the clustering analysis. Branches of the dendrogram group together hub genes that are positively correlated. (C) Eigengene adjacency 
heatmap between modules. Each column and row corresponds to one module hub gene (labeled by color) or weight. In the heatmap, red represents high adjacency (positive correlation), while blue color represents low adjacency (negative correlation). Squares of red color along the diagonal are the metamodule. (D) Scatterplots showing module membership versus gene significance for the green module.

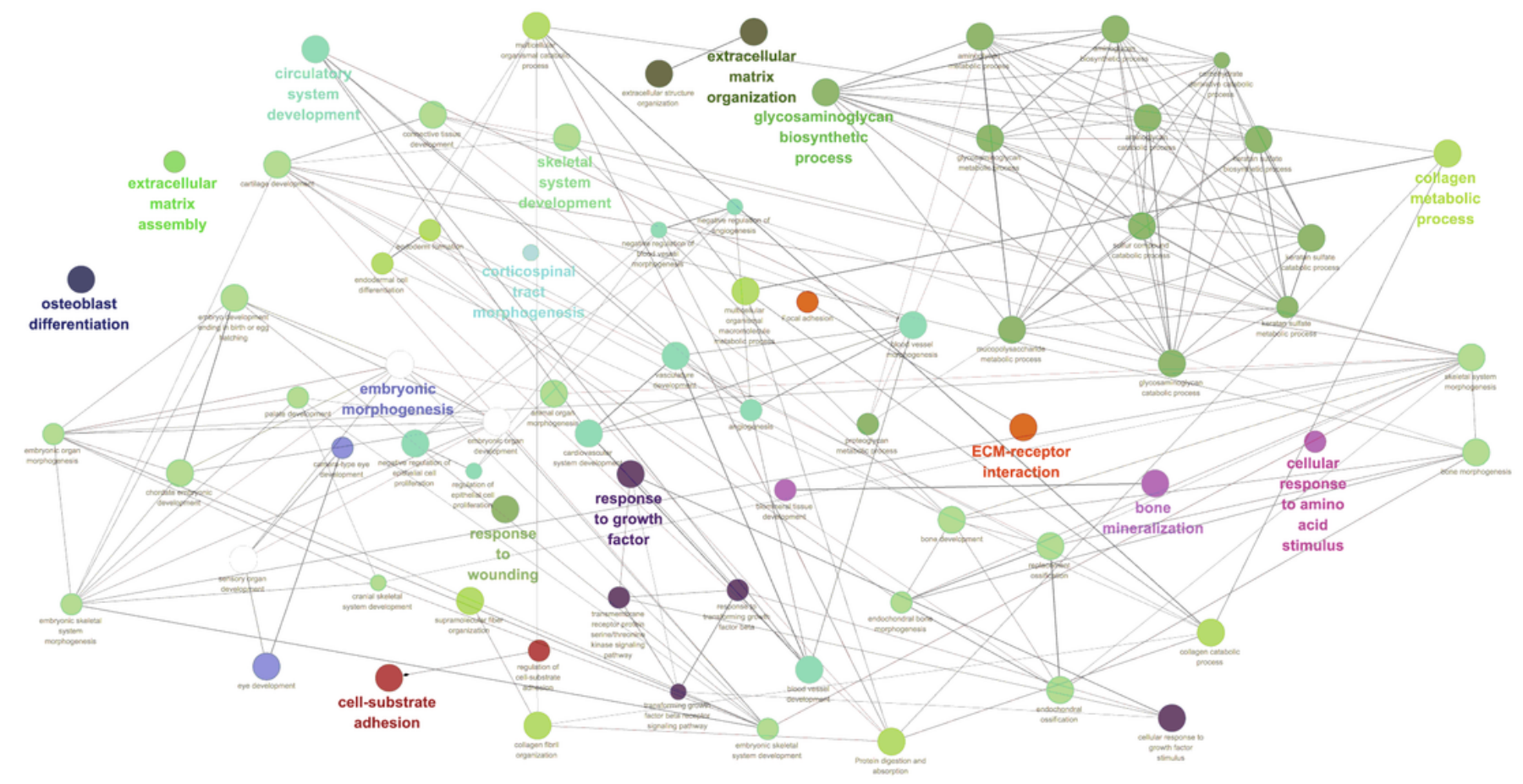

\section{Figure 4}

The cytoscape ClueGO plugin was used to identify enriched KEGG pathways amongst all statistically significant proteins in the fasted and refed comparisons. Nodes containing proteins from related KEGG terms are colored the same, with node size indicating the associated Benjamini-Hochberg corrected $\mathrm{P}$ value for enrichment (smaller $P$ values are indicated by larger node size), and line width indicating the level of overlap between related nodes (thicker lines indicate a greater overlap) 


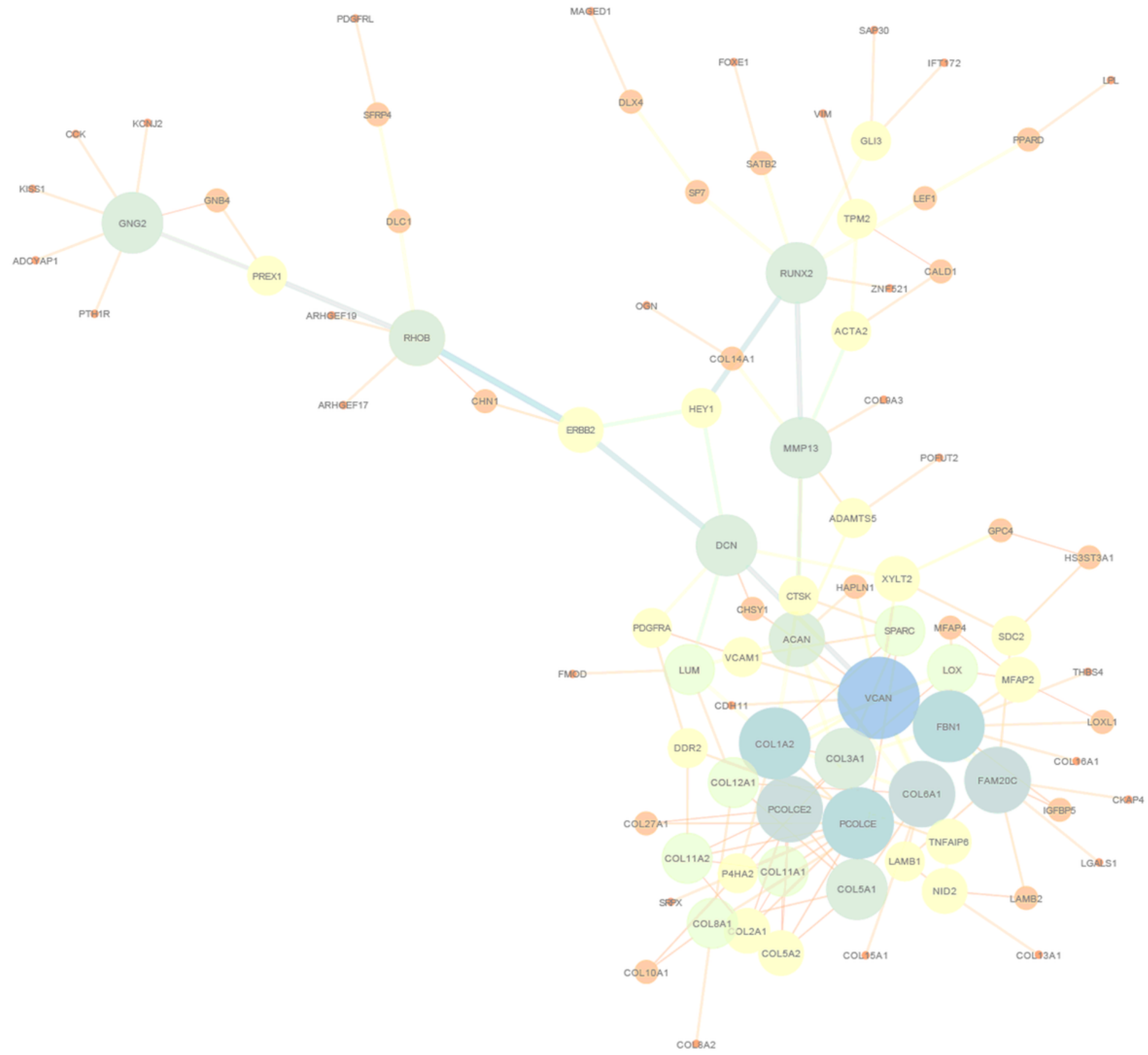

Figure 5

Protein-protein interaction (PPI) network of genes in the green module. The density of the ellipse corresponds to module membership (ME) values. The network was constructed using Cytoscape 3.4 software. 
A

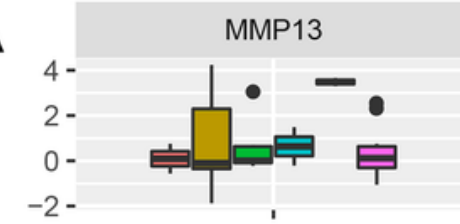

$1.0-$

0.5 -

$0.0-$

-0.5 -

-1.0 -
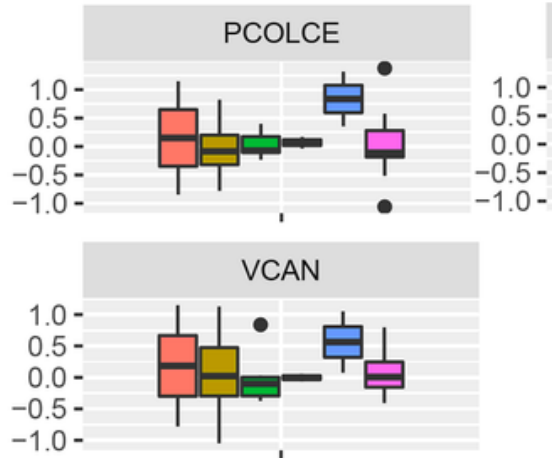

B

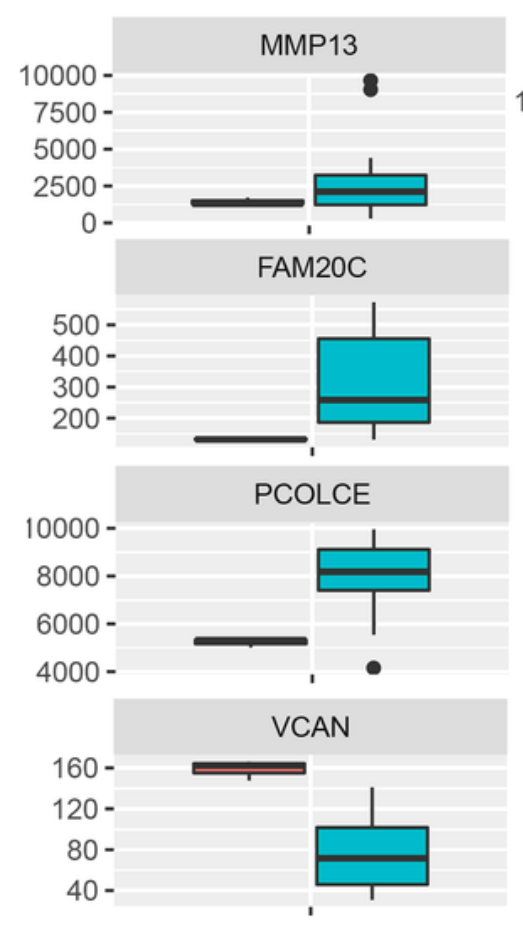

FAM20C
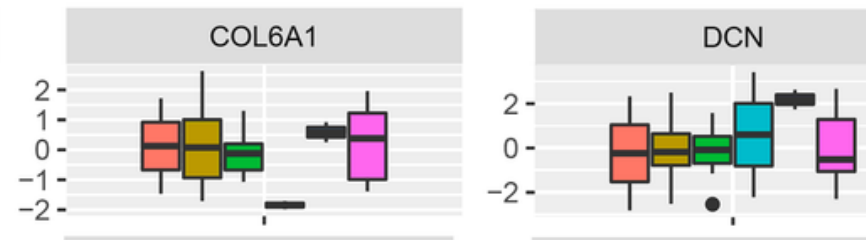

FBN1
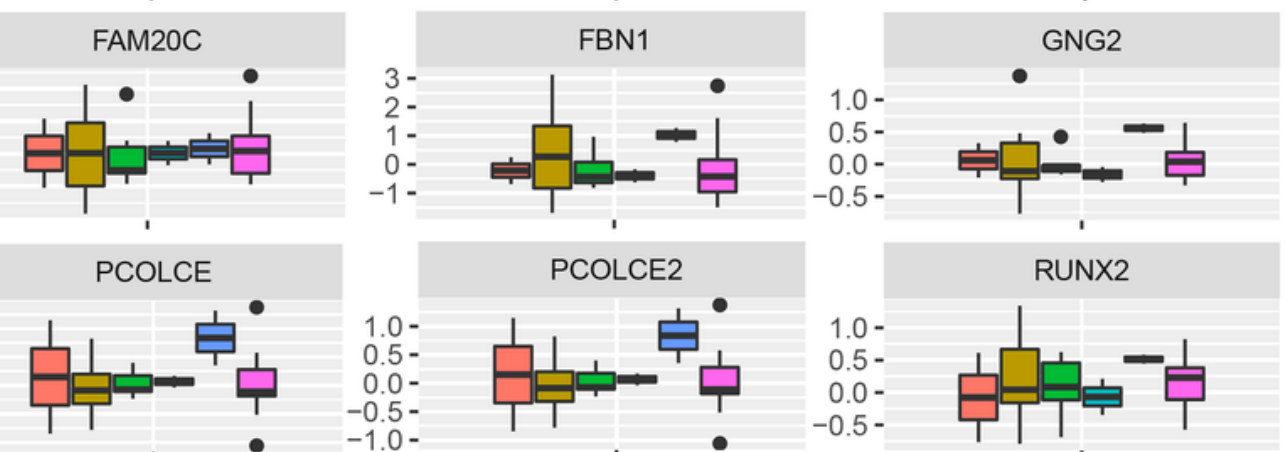

RUNX2

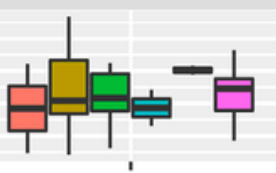

group

舁 calcaneum

它 femur

pelvis

留 rib

包 sacrum

声 tibia

\section{Expression_level}
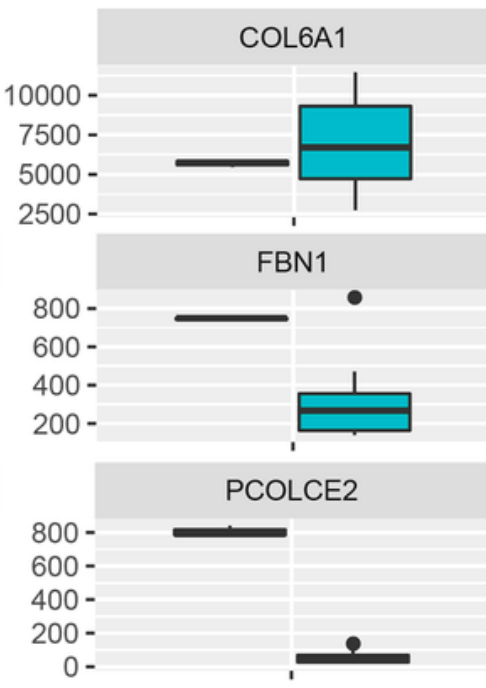

\section{Expression_level}

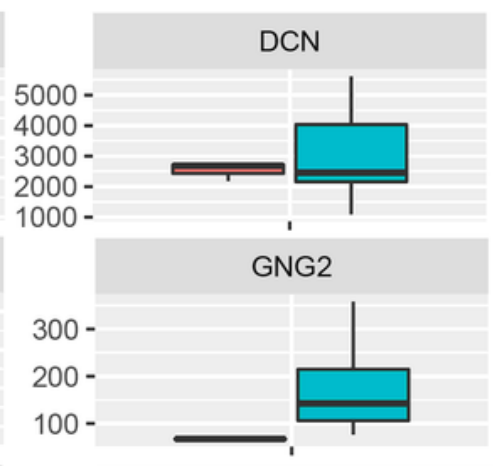

group

它 ctrl

声 tumor

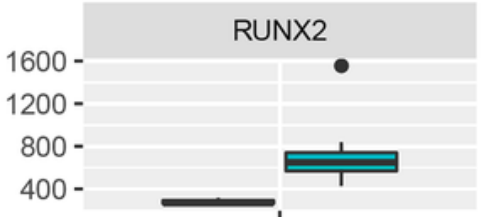

\section{Figure 6}

Validation of hub gene expression. (A) The expression of hub genes in OS samples for GSE19276 training set. (B) The expression of hub genes in 15 OS samples and 3 healthy controls for GSE9460 validation set. 